Tatiana Witjas, MD

Romain Carron, MD,

$\mathrm{PhD}$

Paul Krack, MD, PhD

Alexandre Eusebio, MD,

$\mathrm{PhD}$

Marianne Vaugoyeau,

$\mathrm{PhD}$

Marwan Hariz, MD, PhD

Jean Philippe Azulay,

$\mathrm{MD}, \mathrm{PhD}$

Jean Régis, MD

Correspondence to

Dr. Witjas:

tatiana.witjas@ap-hm.fr

Supplemental data at Neurology.org

\title{
A prospective single-blind study of Gamma Knife thalamotomy for tremor
}

$\underline{\underline{\underline{\underline{E}}}}$

ABSTRACT

Objective: To evaluate the safety and efficacy of unilateral Gamma Knife thalamotomy (GKT) for treatment of severe tremor with a prospective blinded assessment.

Methods: Fifty patients (mean age: 74.5 years; 32 men) with severe refractory tremor (36 essential, 14 parkinsonian) were treated with unilateral GKT. Targeting of the ventral intermediate nucleus (Vim) was achieved with Leksell Gamma Knife with a single shot through a 4-mm collimator helmet. The prescription dose was $130 \mathrm{~Gy}$. Neurologic and neuropsychological assessments including a single-blinded video assessment of the tremor severity performed by a movement disorders neurologist from another center were performed before and 12 months after treatment. MRI follow-up occurred at 3, 6, and 12 months.

Results: The upper limb tremor score improved by $54.2 \%$ on the blinded assessment ( $p<$ 0.0001). All tremor components (rest, postural, and intention) were improved. Activities of daily living were improved by $72.2 \%$. Cognitive functions remained unchanged. Following GKT, the median delay of improvement was 5.3 months (range 1-12 months). The only side effect was a transient hemiparesis associated with excessive edema around the thalamotomy in one patient.

Conclusion: This blinded prospective assessment demonstrates that unilateral GKT is a safe and efficient procedure for severe medically refractory tremor. Side effects were rare and transient in this study.

Classification of evidence: This study provides Class IV evidence that for patients with severe refractory tremor, GKT is well tolerated and effective in reducing tremor impairment. Neurology ${ }^{\circledast 2} 2015 ; 85: 1562-1568$

\section{GLOSSARY}

AC-PC = anterior commissure-posterior commissure; $\mathbf{A D L}=$ activities of daily living; $\mathbf{D B S}=$ deep brain stimulation; $\mathbf{E T}=$ essential tremor; GK = Gamma Knife; GKT = Gamma Knife thalamotomy; IP = improved patient; NIP = nonimproved patient; PD = Parkinson disease; RFT = radiofrequency thermocoagulation; Vim = ventral intermediate nucleus.

The pharmacologic treatment for severe tremor can be disappointing. ${ }^{1,2}$ Stereotactic surgery, such as radiofrequency thermocoagulation (RFT) or deep brain stimulation (DBS), may be a therapeutic option. ${ }^{3-5}$ DBS has supplanted RFT as the reference technique because of its efficacy, potential reversibility, adaptability, and safety even when performed bilaterally. ${ }^{2,6-11}$ However, both RFT and DBS may carry significant risks including intracerebral hemorrhage, infection, and hardware-related complications. Both techniques may also be contraindicated in some conditions (anticoagulant medication, comorbidities, or very advanced age).

Gamma Knife (GK) radiosurgery is a minimally invasive neurosurgical procedure without craniotomy and therefore no risk of intracranial infection or bleeding. Since the first 1992 publication, ${ }^{12}$ several studies ${ }^{13-17}$ have reported the efficacy of GK thalamotomy (GKT) on severe tremor (essential tremor [ET] and tremor-dominant Parkinson disease [PD]) with a low rate of complications. However, as highlighted in a recent review, ${ }^{18}$ the level of evidence is weak and

\footnotetext{
From the Department of Neurology and Movement Disorders (T.W., A.E., J.P.A.) and Department of Functional and Stereotactic Neurosurgery and Radiosurgery (R.C., J.R.), Assistance Publique-Hôpitaux de Marseille, Aix-Marseille Université, Timone University Hospital; Institut de Neurosciences de la Timone (T.W., A.E., J.P.A.), UMR 7289, CNRS Aix-Marseille Université, Marseille, France; Université Grenoble Alpes (P.K.), GIN INSERM U318 and CHU de Grenoble, Movement Disorder Unit, Grenoble, France; CNRS-Aix-Marseille Université (M.V.), Laboratory of Cognitive Neurosciences, FR 3 C, Marseille, France; Unit of Functional Neurosurgery (M.H.), UCL Institute of Neurology, Queen Square, London, UK; Department of Clinical Neuroscience (M.H.), Umeå University, Sweden; and INSERM (R.C., J.R.), UMR 1106, Aix-Marseille Université, Marseille, France. Go to Neurology.org for full disclosures. Funding information and disclosures deemed relevant by the authors, if any, are provided at the end of the article.
} 
suffers from being mainly based on retrospective cohort studies, a single prospective study with no independent evaluation, and only one single-blind study on 14 patients. ${ }^{13-17,19}$ These studies comprise significant flaws. ${ }^{2,18}$ An accurate independent and blinded assessment of tremor control is a critical step in determining whether the procedure is successful.

The aim of this study was to prospectively assess the efficacy of unilateral GKT in a large group of patients with severe tremor using blinded video assessments, in addition to the evaluation of activities of daily living (ADL), neuropsychological testing, and neuroimaging assessments.

METHODS The study was designed to provide Class IV evidence that for patients with severe refractory tremor, GKT is well tolerated and effective in reducing tremor. Between September 2004 and September 2010, 61 consecutive patients, referred to Timone University Hospital in Marseille from several French Movement Disorders departments, were prospectively evaluated and underwent unilateral GKT for treatment of medically refractory essential or parkinsonian tremor. Patients were included if they had severe disabling tremor of one or both upper limbs, if they could not undergo Vim DBS for any medical or surgical reasons, or if they were refusing DBS. Patients younger than 18 years, patients unable to fully understand and give their consent to the therapeutic proposal because of cognitive or psychiatric conditions, and patients with abnormal anatomy of their thalamic area were excluded. There was no maximum age limitation.

Eleven patients were unable to complete the 12-month follow-up (7 patients were lost to follow-up, 3 were hospitalized for concomitant disease, and one refused the last video assessment) and were excluded from the main analysis. The main difficulties for follow-up were the advanced age of the patients and

\begin{tabular}{|c|c|}
\hline Patient characteristics & \\
\hline No. of patients & 50 \\
\hline Male/female & $32 / 18$ \\
\hline Handedness & $46 \mathrm{R} / 4 \mathrm{~L}$ \\
\hline Age at GK treatment, $y$ & $74.5(60-91)^{\mathrm{a}}$ \\
\hline Disease duration, $y$ & $22.4(4-74)^{a}$ \\
\hline Etiology of tremor & $36 \mathrm{ET} / 14 \mathrm{PD}$ \\
\hline Treated Vim & $\begin{array}{l}38 \text { left } \operatorname{Vim} / 12 \\
\text { right } \operatorname{Vim}\end{array}$ \\
\hline \multicolumn{2}{|l|}{$\begin{array}{l}\text { Major factors in the decision to use } \\
\text { GK thalamotomy rather than DBS }\end{array}$} \\
\hline Medical comorbidities & 25 \\
\hline Advanced age (> 75 y) & 17 \\
\hline Patient refusal of DBS (age $<75 \mathrm{y}$ ) & 8 \\
\hline
\end{tabular}

Abbreviations: DBS = deep brain stimulation; $E T=$ essential tremor; PD = Parkinson disease; Vim = ventral intermediate nucleus.

${ }^{\text {a }}$ Range. the fact that many patients (41\%) lived far away from Marseille. Some follow-up data could be retrieved for 9 of these patients (medical records, telephone call, etc.); table e-1 on the Neurology ${ }^{\circledR}$ Web site at Neurology.org gives the details on this informal follow-up.

Two patients with PD had previously (12 and 15 years earlier) undergone unilateral Vim DBS. With the emergence of severe contralateral upper limb tremor, a GKT was proposed, their advanced age contraindicating a contralateral DBS lead implantation. One patient with ET and bilateral Vim DBS had left GKT after leads were removed due to hardware infection. Demographic data and clinical characteristics of all patients are shown in table 1 .

Assessments were prospectively performed before GKT and at 6 and 12 months following radiosurgery. Tremor severity of the upper limbs was assessed according to the Fahn-Tolosa-Marin Tremor Rating Scale ${ }^{20}$ and item "drinking from a cup" from the Washington Heights-Inwood Genetic Study of Essential Tremor Rating Scale. ${ }^{21}$ The Tremor Rating Scale items included resting, postural, and action/intention tremor using the finger-tonose test (for each of these items, scores ranged from $0=$ no tremor to $4=$ severe tremor, with amplitude $>4 \mathrm{~cm}$ ); handwriting $(0=$ normal; $4=$ severely abnormal, unable to keep pen on paper without holding the hand down); drawing (Archimedes spirals A large); and pouring water from one cup to another $(0=$ normal, $4=$ unable to pour water without spilling most of it) and drinking from a cup ( $0=$ normal, $4=$ unable to hold the cup). The global tremor score was calculated as the sum of the above-mentioned items (total score $=28$ if writing was considered, 24 if not). For items in which both sides were scored, only the side contralateral to the thalamotomy was taken into account.

The tremor severity was assessed without interrupting the medication. The assessments were performed by the same neurologist (T.W.) who did the video recordings for the 50 patients. ADLs were assessed using a self-questionnaire designed by Bain ( 25 items, score 1: able to do the activity without difficulty; 4 : cannot do the activity). ${ }^{22}$ Cognitive performances were assessed using Mattis Dementia Rating Scale and verbal fluency test. Five patients were not able to complete a full neuropsychological testing because of language barrier (2 patients), severe upper limb and voice tremor (2 patients), and refusal to do the assessment at 12 months (1 patient).

An independent movement disorders neurologist (P.K.) assessed the tremor severity score by viewing videos of each patient before and at 1 year after unilateral GKT in a randomized, blinded manner concerning both the laterality of the thalamotomy and the state of the patient (before or after treatment). The video-based tremor evaluation scored the following items (from 0 to 4): rest, postural (both arms outstretched), intention (finger to nose), Archimedes spiral drawing, handwriting, drinking, and pouring a glass of water (total score on 28). The tremor score was performed only in the upper limbs. Blinded assessment of voice, head, or lower limb action tremor on walking was not analyzed because unilateral GKT is known to have little or no effect on axial symptoms. Moreover, these features are more difficult to rate reliably based on video assessment and the upper limb tremor was the predominant symptom in all the patients.

Serial MRIs were obtained at 3, 6, and 12 months after radiosurgery. The radiosurgical procedures were all performed by the same neurosurgeon (J.R.). Patients, in agreement with their choice, were unilaterally treated on the contralateral side of the most disabled limb. The Leksell $\mathrm{G}$ frame was applied under local anesthesia. Special care was taken to place the stereotactic frame parallel to the orientation of the anterior commissure-posterior commissure (AC-PC) line and to minimize head tilt or rotation. 
Stereotactic imaging consisted of an axial T2-weighted halfmillimetric acquisition (CISS [Constructive Interference in Steady State], 1.5T; Siemens, Erlangen, Germany) at the level of AC-PC providing a magnetic resonance "ventriculography" of the third ventricle, a turbo spin-echo coronal $3 \mathrm{~mm}$ thick at the level of thalamus, and a T1-weighted 3-dimensional acquisition (magnetization-prepared rapid-acquisition gradient echo,1.5T; Siemens). An additional stereotactic CT scan was systematically performed and coregistered to rule out any potential distortion of the MRI scans.

Treatment planning was performed with Leksell Gamma Plan software (8.3.1 up to 10.1.1 version) (Elekta, Stockholm, Sweden). Nonstereotactic images, including diffusion tensor images acquired before the treatment, were fused with the stereotactic ones. AC and PC were defined on the magnetic resonance ventriculography. The center of the target, determined according to Guiot's diagram, was placed $2.5 \mathrm{~mm}$ above the AC-PC line, 11 $\mathrm{mm}$ lateral to the wall of the third ventricle, and at a mean of $7.3 \mathrm{~mm}$ in front of the PC (3.9-9.9; SD: 1.33).

A unique 4-mm isocenter was used, as well as beam blocking to optimize the gradient of dose toward the internal capsule. The prescription dose was 130 Gy (maximum dose) in all patients. The patient was discharged the day after the radiosurgical procedure. The evolution of the GK radiosurgical lesion within the Vim was analyzed for all the patients by the same neurosurgeon (R.C.) who was blinded to the clinical results.

Descriptive statistics are reported in the text as median and interquartile interval in parenthesis (Q3-Q1) except the results for each subscore of the tremor (mean \pm SEM). To explore the GK effect, the comparisons between scores before and at 12 months after GK were tested using Wilcoxon signed rank test. The percentage of improvement both for tremor severity and ADL was obtained based on the difference between these scores assessed at baseline and at 1 year after radiosurgery. Weighted $\kappa$ was used to assess interrater reliability measured for both global and subscores. ${ }^{23}$ The relationship between the ADL improvement and the symptomatic amelioration rates was investigated using the Spearman correlation. Differences with a $p$ value $<0.05$ were considered to be statistically significant.

Standard protocol approvals, registrations, and patient consents. All patients participated with informed written consent. The study was allowed by the local ethics committee, and was in compliance with the national legislation and the Declaration of Helsinki.

RESULTS Medians and interquartile intervals of the tremor score improvement are presented in figure 1 for both assessments, blinded and unblinded. The unblinded assessment showed an improvement of the global tremor score by $63.40 \%$ (38.3-90.6) ( $W=$ $1,248, p<0.0001)$ as well as an improvement of each subscore. The blinded assessment demonstrated an improvement in the upper limb tremor score by $54.2 \%(36.2-79.2)(W=1,159, p<0.0001)$ as well as an improvement of each subscore (figure 2 ). The unblinded assessment of the ipsilateral limb and axial features showed no significant improvement.

Interrater reliability. The $\kappa$ coefficient for the total score between blinded and unblinded assessments was 0.74 (95\% confidence interval $0.66-0.82)$ before the treatment and $0.78(95 \%$ confidence interval
Figure 1 Pre- and post-GK comparison

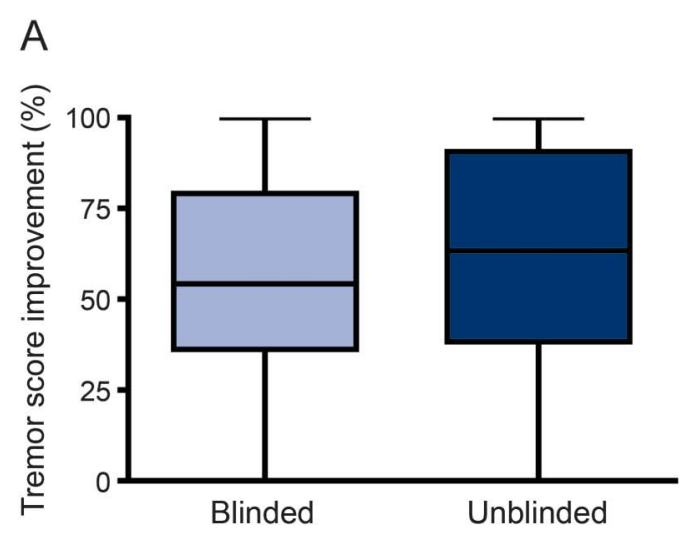

B

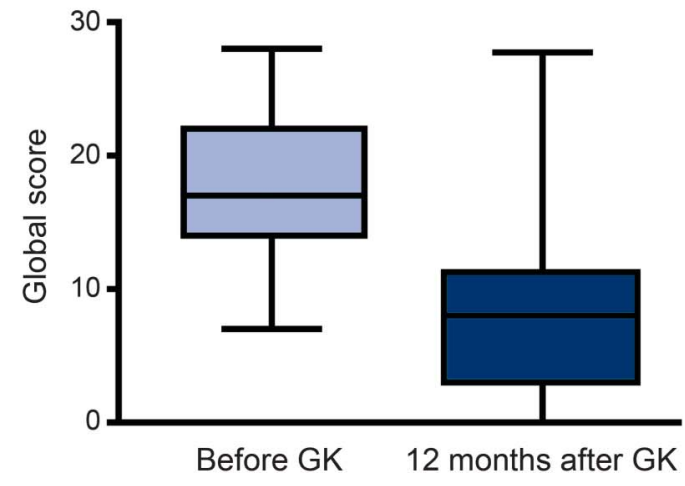

(A) Tremor score improvement for the blinded and unblinded assessments. (B) Effect of treatment. The boxes extend from the first to third quartiles. The lines in the middle represent the medians. The whiskers go down to the smallest value and up to the largest. $* * * p<0.001$. After unilateral GK thalamotomy, there was a significant improvement in the upper limb tremor score whatever the item considered. $\mathrm{GK}=$ Gamma Knife.

0.72-0.85) 12 months after unilateral GKT. These results indicate in both cases a good interrater reliability. ${ }^{23}$ Globally for all subscores, before as well as after GKT, the statistical analysis revealed a good strength of agreement between both raters. Therefore, all tremor scores used hereafter are based on the blinded assessment.

The mean improvement in ADL was $72.2 \%(0 \%-$ $100 \%)$. To assess whether the ADL improvement was a good index to evaluate the treatment effect, we correlated the ADL improvement with the global tremor score and subscore improvements according to the blinded assessment. We found that the tremor score improvement was correlated with the ADL improvement $(r=0.68 ; p<0.0001)$. To determine the functional outcome of GKT, we divided the cohort into 2 groups according to $\mathrm{ADL}$ improvement: improved patients (IPs) ( $\geq 50 \%)$ and nonimproved 


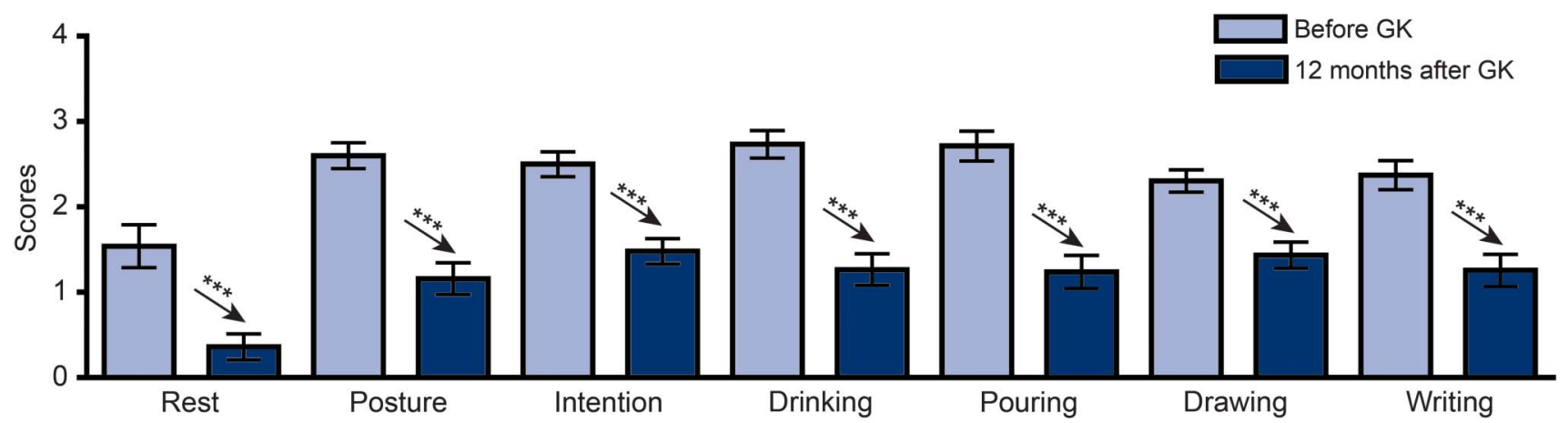

Mean and SEM of each subscore. ${ }^{* * *} p<0.001$. All subscores improved after radiosurgery as compared with before radiosurgery for the blinded rater. GK $=$ Gamma Knife.

patients (NIPs) (<50\%). We found that 38 patients were improved (median ADL improvement $96.4 \%$ [87.5\%-100\%]) and 12 were nonimproved (median ADL improvement $0 \%[5.9 \%-15.2 \%])$. These 38 IPs improved their global tremor score by $64.9 \%$ (range 50\%-86\%). It is noteworthy that before GK treatment, there was no difference in tremor severity between the 2 groups (median 21 [14.8-25] and 17 [14-22] for NIPs and IPs, respectively). Neither the tremor etiology (PD or ET) nor the tremor score severity influenced the outcome.

Of the 9 patients lost to follow-up for whom some follow-up data could be retrieved, the tremor was deemed significantly reduced for 5 (table e-1).

The delay before any improvement could be identified by the patient varied usually between 4 and 6 months, with a range of 1 to 12 months (median 5.3) after radiosurgery.

The neuropsychological assessment did not reveal any significant change in Mattis Dementia Rating Scale: 135/144 (132-137) pre-GKT and 134 (131139) 12 months after GKT. There was a slight nonsignificant decrease in literal fluency (15 [11-17] vs 11 [8-17.3]) but a significant decrease in semantic fluency (25 [19.5-30] vs 22 [17-28]; $W=169 ; p<$ 0.0252).

Concerning side effects, one patient developed a hemiparesis contralaterally to the thalamotomy at 12 months. This motor deficit regressed and the patient recovered spontaneously within 3 weeks without any neurologic sequelae and without any steroid prescription. He had no tremor afterward. The $2 \mathrm{pa}-$ tients with PD and previous DBS contralateral to GKT did not experience any adverse event due to the procedure. The patient with ET who had previous bilateral DBS had an improvement of his tremor score by $45.7 \%$ after left GKT. His ADLs were improved by $66.7 \%$. Before the stimulation lead was removed due to infection, he reported an $85 \%$ improvement of his tremor score with DBS.
Imaging follow-up analysis. Thirty-eight of the $50 \mathrm{pa}-$ tients had a normal neuroimaging pattern of response defined as T2-weighted and fluidattenuated inversion recovery hyperintense limited signal changes and a postcontrast ring-shaped lesion at 12 months (figures 3 and e-1A). One patient had an excessive response (magnetic resonance scan showing an abnormally large area of enhancement with massive edema extending beyond the internal capsule) that resolved thereafter (figure e-1B). For 11 patients, imaging analysis showed either the absence of visible lesion or very minimal alterations. All 38 clinically improved patients had a normal (or hyper for one) neuroimaging pattern. Of the 12 NIPs, one had a normal radiologic response with correct stereotactic targeting whereas 11 patients displayed no or minimal changes (figure e-1C).

DISCUSSION The present prospective study with an independent and single-blinded video assessment of 50 patients, mean age of 75 years (60-91), with severe essential $(n=36)$ or PD-related $(n=14)$ tremor demonstrates that GKT safely and effectively reduces disabling upper limb tremor. The global tremor score improved by $54.2 \%$ on blinded evaluation and ADL by $72.2 \% \quad 1$ year after unilateral thalamotomy. ADL improved by $50 \%$ or more in 38 patients $(76 \%)$, and the improvement of the global tremor score in these patients was $64.9 \%$. These results are in line with previous studies on GKT for severe tremor. ${ }^{14,15,17}$ The experience of GK radiosurgery for movement disorders started as early as the 1960s with 470 procedures reported in more than 70 studies to date. Most of them reported good efficacy on tremor $(60 \%-90 \%$ improvement) with a low rate of side effects $(<8 \%))^{14,15,17}$ The positive effect of GKT is sustained over time. ${ }^{15}$ However, these data were criticized because they were mostly uncontrolled case series. ${ }^{18}$ The only study with a blinded assessment was conducted on 

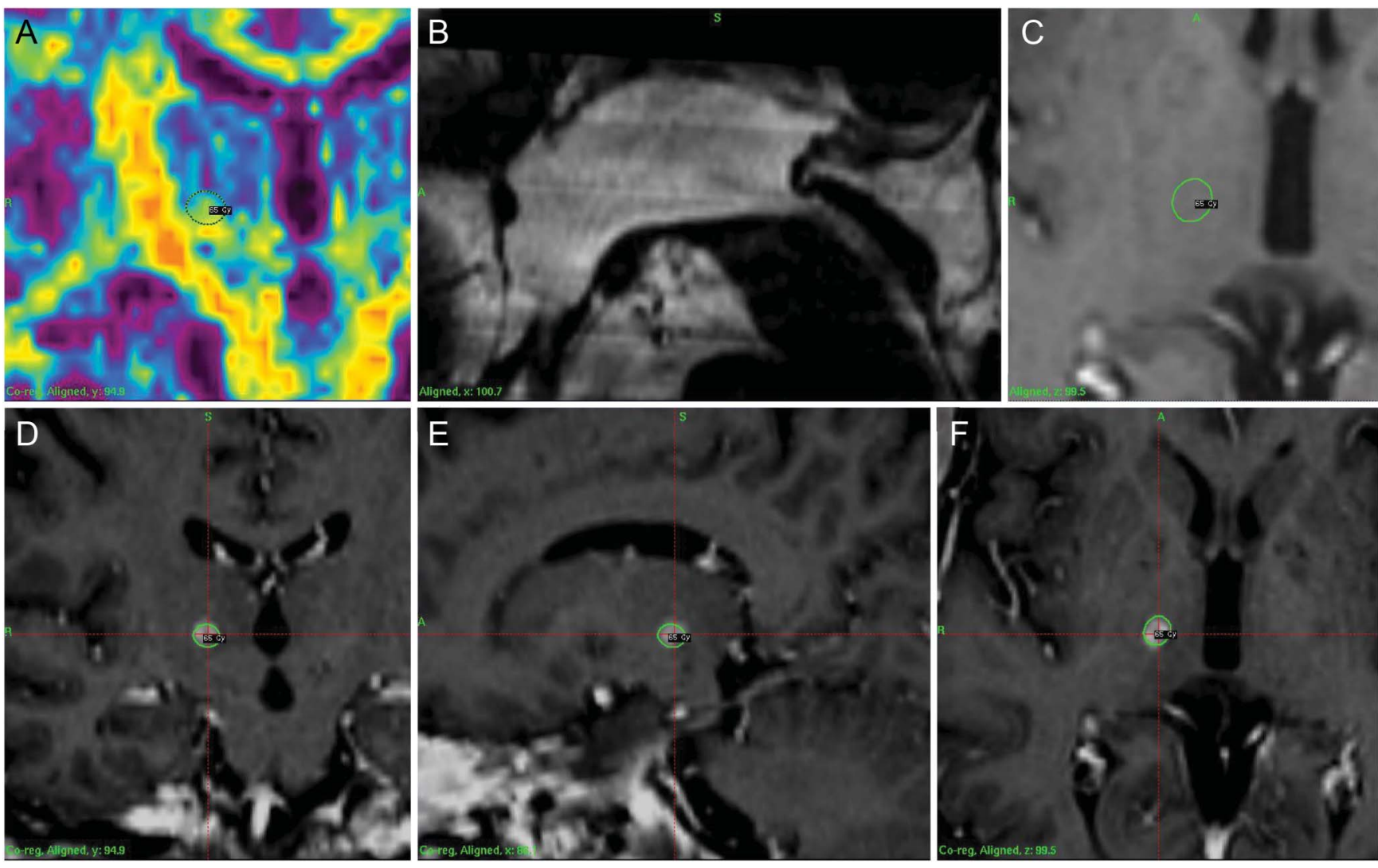

The thalamic lesion matches exactly with the planned target (as outlined by the 65-Gy [50\%] isodose line). Upper panels: Diffusion tensor imaging coronal (A), T2-sagittal (B), and gadolinium-enhanced T1-weighted axial (C). Lower panels: Gadolinium-enhanced T1-weighted in coronal (D), sagittal (E), and axial (F) planes.

14 patients with a variable follow-up (range 7-30 months). ${ }^{19}$ The effect of GKT in this series was in stark contrast to the rest of the literature. The authors reported no significant tremor reduction but an improvement in ADL. However, the follow-up $(<1$ year) for some patients was probably too short to conclude for inefficacy. In our experience, the delay of efficacy can be as long as 1 year as previously reported. The discrepancy between this series and the rest of the literature has emphasized the need for a prospective large cohort study with an independent and blinded assessment.

GKT is less invasive than open skull neurosurgical procedures. Adverse events consist of motor deficit, paraesthesia, or dysarthria with a rate ranging from $1.6 \%$ to $8.4 \%$. In a study with blinded assessments, the authors ${ }^{19}$ reported 3 adverse events: 2 mild (nondisabling lip and finger numbness at 18 and 19 months) and one severe (thalamic hemorrhage at 14 months). In the present study, only one patient (2\%) had a transient side effect, consisting of hemiparesis, associated with marked alterations on the MRI. The deficit resolved spontaneously within 3 weeks. This side effect was the consequence of a hyperresponse to radiosurgery. A Japanese study has reported that approximately $10 \%$ of the patients may have a hyperresponse upon imaging with associated clinical side effects. ${ }^{14}$ However, these side effects are reported to be transient in most cases with no or minimal sequelae. ${ }^{17}$ Eleven patients (18\%) could not complete the follow-up. This relatively high dropout rate may be explained by the fact that patients were referred from centers all over France and this elderly population with comorbidities was not always able to travel. However, some follow-up data could be retrieved for 9 patients. Although this is a limitation inherent to the study design, we believe that the subjective assessments obtained indicate that most of these patients also improved.

One challenge with GKT is the variability of the thalamic reaction. In this study, we showed that all IPs had a lesion within the Vim that matches exactly with the radiosurgical planning. However, our results also showed, to our knowledge for the first time, that all of the NIPs but one had no or minimal lesions on MRI, corresponding to a hyporesponse pattern. This individual variability in the thalamic reactions to radiosurgery, although the procedure was strictly performed in the same conditions, will need further investigation. 
Several surgical strategies have been proposed over the past decades for severe medically refractory tremor and have transformed the outcome of this severely disabling condition. These include radiofrequency thermo-thalamotomy, DBS, and GK radiosurgery. The advantage of DBS is that it can be performed bilaterally. Today, thermo-thalamotomies are performed unilaterally either by radiofrequency or LITT (laser-induced thermal therapy) or HIFU (highintensity focused ultrasound). Both LITT and HIFU are methods for a very early stage and require intraoperative (MRI) temperature monitoring. These 2 techniques are still investigational with limited experience in the literature and a lack of blinded assessment and long-term evaluation. ${ }^{24-26}$

The radiobiological effect of radiosurgery is unique. This delayed biological effect is completely different from the acute physical thermocoagulation effect. The circumscribed central necrotic lesion (4-mm diameter) where the highest dose was delivered is surrounded by a nonnecrotic halo consisting of vascular changes and astrocytogliosis. ${ }^{27,28}$ These peripheral modifications may induce a larger therapeutic effect than thermolesions. ${ }^{28}$ This peripheral effect may explain the sustained beneficial effect after GKT reported in most studies. ${ }^{15,17}$ The very small size of the residual lesion on long-term follow-up has led some authors to discuss the possibility of a nonlesional effect of radiosurgery around the small necrotic scar. $^{27,28}$

This single-blind prospective study showed that unilateral GKT allows a significant benefit for patients with medically refractory upper limb tremor with both a reduction of the tremor score and an improvement in $\mathrm{ADL}$. The adverse events are rare (2\%) and usually transient. Long-term effects of GKT in the present cohort will need to be examined in follow-up studies. Future randomized studies comparing risk-benefit ratios for the different existing surgical techniques from DBS to ablative surgery for disabling medication-resistant tremors are warranted. The present study shows that GKT may provide a unique opportunity to improve ADL in a population of elderly patients with disabling tremor or patients not suitable to undergo a more invasive procedure..$^{29}$

\section{AUTHOR CONTRIBUTIONS}

Tatiana Witjas: drafting/revising the manuscript, study concept or design, analysis or interpretation of data, accepts responsibility for conduct of research and will give final approval, contribution of vital reagents/ tools/patients, acquisition of data, statistical analysis, study supervision. Romain Carron: drafting/revising the manuscript, analysis or interpretation of data, accepts responsibility for conduct of research and will give final approval, acquisition of data. Paul Krack: drafting/revising the manuscript, analysis or interpretation of data, accepts responsibility for conduct of research and will give final approval. Alexandre Eusebio: drafting/revising the manuscript, analysis or interpretation of data, accepts responsibility for conduct of research and will give final approval, acquisition of data. Marianne Vaugoyeau: analysis or interpretation of data, accepts responsibility for conduct of research and will give final approval, statistical analysis. Marwan Hariz: drafting/revising the manuscript, analysis or interpretation of data, accepts responsibility for conduct of research and will give final approval. Jean Philippe Azulay: drafting/revising the manuscript, study concept or design, analysis or interpretation of data, accepts responsibility for conduct of research and will give final approval, acquisition of data, study supervision. Jean Régis: drafting/revising the manuscript, study concept or design, analysis or interpretation of data, accepts responsibility for conduct of research and will give final approval, acquisition of data, study supervision, obtaining funding.

\section{STUDY FUNDING}

No targeted funding reported.

\section{DISCLOSURE}

T. Witjas has received honoraria from UCB, AbbVie, Teva, and Medtronic and has received research grant from the French Ministry of Health, all unrelated to the present study. R. Carron has received grants for congress participations from Medtronic and Cyberonics, both unrelated to the present study. P. Krack has received honoraria for lecturing or consultation from the Movement Disorder Society, Lundbeck, Boehringer Ingelheim, Novartis, UCB, Medtronic, Orkyn, Abbott, Orion, Teva, and Boston Scientific, and research support from the French Ministry of Health (PHRC), France Parkinson, INSERM (French National Institute of Health and Research in Medicine), Grenoble University Hospital, Orkyn, Novartis, Medtronic, LVL, and St. Jude, all of which are unrelated to the present study. A. Eusebio has received honoraria from UCB, GE Healthcare, and Aguettant, is on the advisory board for Orkyn, and has received research grants from the French Ministry of Health and the Agence Nationale de la Recherche, all unrelated to the present study. M. Vaugoyeau reports no disclosures relevant to the manuscript. M. Hariz has received honoraria and travel expenses from Medtronic and St. Jude for speaking at meetings, both unrelated to the present study. J. Azulay has received honoraria from AbbVie, Boehringer, and UCB and is in the advisory board for AbbVie, Zambon, and UCB, all unrelated to the present study. J. Régis has received honoraria from Medtronic and a research grant from Elekta, both unrelated to the present study. Go to Neurology.org for full disclosures.

Received March 8, 2015. Accepted in final form July 6, 2015.

\section{REFERENCES}

1. Elias WJ, Shah BB. Tremor. JAMA 2014;311:948-954.

2. Zesiewicz TA, Elble R, Louis ED, et al. Practice parameter: therapies for essential tremor: report of the Quality Standards Subcommittee of the American Academy of Neurology. Neurology 2005;64:2008-2020.

3. Ohye $\mathrm{C}$, Narabayashi $\mathrm{H}$. Physiological understanding of the thalamic ventralis intermedius nucleus [proceedings]. Appl Neurophysiol 1979;42:312.

4. Tasker RR, Siqueira J, Hawrylyshyn P, Organ LW. What happened to VIM thalamotomy for Parkinson's disease? Appl Neurophysiol 1983;46:68-83.

5. Narabayashi H, Maeda T, Yokochi F. Long-term followup study of nucleus ventralis intermedius and ventrolateralis thalamotomy using a microelectrode technique in parkinsonism. Appl Neurophysiol 1987;50:330-337.

6. Tasker RR. Deep brain stimulation is preferable to thalamotomy for tremor suppression. Surg Neurol 1998;49: 145-153; discussion 153-154.

7. Schuurman PR, Bosch DA, Bossuyt PM, et al. A comparison of continuous thalamic stimulation and thalamotomy for suppression of severe tremor. N Engl J Med 2000;342:461-468.

8. Schuurman PR, Bosch DA, Merkus MP, Speelman JD. Longterm follow-up of thalamic stimulation versus thalamotomy for tremor suppression. Mov Disord 2008;23:1146-1153. 
9. Benabid AL, Pollak P, Gao D, et al. Chronic electrical stimulation of the ventralis intermedius nucleus of the thalamus as a treatment of movement disorders. J Neurosurg 1996; 84:203-214.

10. Hariz MI, Krack P, Alesch F, et al. Multicentre European study of thalamic stimulation for parkinsonian tremor: a 6 year follow-up. J Neurol Neurosurg Psychiatry 2008;79: 694-699.

11. Flora ED, Perera CL, Cameron AL, Maddern GJ. Deep brain stimulation for essential tremor: a systematic review. Mov Disord 2010;25:1550-1559.

12. Lindquist C, Steiner L, Hindmarsh T. Gamma Knife thalamotomy for tremor: report of two cases. In: Steiner L, Lindquist C, Forster D, editors. Radiosurgery: Baseline and Trends. New York: Raven Press; 1992:237-243.

13. Niranjan A, Kondziolka D, Baser S, Heyman R, Lunsford LD. Functional outcomes after Gamma Knife thalamotomy for essential tremor and MS-related tremor Neurology 2000;55:443-446.

14. Ohye C, Higuchi Y, Shibazaki T, et al. Gamma Knife thalamotomy for Parkinson disease and essential tremor: a prospective multicenter study. Neurosurgery 2012;70: 526-535; discussion 535-536.

15. Young RF, Li F, Vermeulen S, Meier R. Gamma Knife thalamotomy for treatment of essential tremor: long-term results. J Neurosurg 2010;112:1311-1317.

16. Kondziolka D, Ong JG, Lee JY, Moore RY, Flickinger JC, Lunsford LD. Gamma Knife thalamotomy for essential tremor. J Neurosurg 2008;108:111-117.

17. Kooshkabadi A, Lunsford LD, Tonetti D, Flickinger JC, Kondziolka D. Gamma Knife thalamotomy for tremor in the magnetic resonance imaging era. J Neurosurg 2013; 118:713-718.

18. Deuschl G, Raethjen J, Hellriegel H, Elble R. Treatment of patients with essential tremor. Lancet Neurol 2011;10: 148-161.
19. Lim SY, Hodaie M, Fallis M, Poon YY, Mazzella F, Moro E. Gamma Knife thalamotomy for disabling tremor: a blinded evaluation. Arch Neurol 2010;67:584-588.

20. Faln S, Tolosa E, Marin C. Clinical rating scale for tremor. In: Jankovic J, Tolosa E, editors. Parkinson's Disease and Movement Disorders. Baltimore: Urban and Schwarzenberg; 1988:225-234.

21. Louis ED, Wendt KJ, Albert SM, Pullman SL, Yu Q, Andrews $\mathrm{H}$. Validity of a performance-based test of function in essential tremor. Arch Neurol 1999;56:841-846.

22. Bain PG, Findley LJ, Atchison P, et al. Assessing tremor severity. J Neurol Neurosurg Psychiatry 1993;56: 868-873.

23. Altman DG, Dore CJ. Baseline comparisons in randomized clinical trials. Stat Med 1991;10:797-799.

24. Elias WJ, Huss D, Voss T, et al. A pilot study of focused ultrasound thalamotomy for essential tremor. $\mathrm{N}$ Engl J Med 2013;369:640-648.

25. Lipsman N, Schwartz ML, Huang Y, et al. MR-guided focused ultrasound thalamotomy for essential tremor: a proof-of-concept study. Lancet Neurol 2013;12:462-468.

26. Moser D, Zadicario E, Schiff G, Jeanmonod D. Measurement of targeting accuracy in focused ultrasound functional neurosurgery. Neurosurg Focus 2012;32:E2.

27. Ohye C, Shibazaki T, Ishihara J, Zhang J. Evaluation of gamma thalamotomy for parkinsonian and other tremors: survival of neurons adjacent to the thalamic lesion after gamma thalamotomy. J Neurosurg 2000;93(suppl 3): 120-127.

28. Regis J, Carron R, Park M. Is radiosurgery a neuromodulation therapy? A 2009 Fabrikant award lecture. J Neurooncol 2010;98:155-162.

29. Hariz GM, Blomstedt P, Koskinen LO. Long-term effect of deep brain stimulation for essential tremor on activities of daily living and health-related quality of life. Acta Neurol Scand 2008;118:387-394.

\section{Minutes Pack a Punch}

\section{Neurology ${ }^{\circledR}$ Podcasts}

- Interviews with top experts on new clinical research in neurology

- Editorial comments on selected articles

- Convenient-listen during your commute, at your desk, or even at the gym

- On demand-it's there when you want it

- Fun and engaging

- New topic each week

- FREE

Listen now at www.aan.com/podcast 